\section{Reversible fibroblast phenotypes in HF}

Human cardiac myofibroblasts in late-stage heart failure (HF) are not terminally differentiated, but actually retain the capacity for dedifferentiation, according to a new study published in JACC. This finding highlights the potential of antifibrotic therapies targeting myofibroblasts to possibly reverse fibrosis in patients with end-stage HF.

The adverse remodelling process in the heart that precedes the onset of $\mathrm{HF}$ involves increased fibrosis, which contributes to both impaired diastolic filling and contractile dysfunction. "Mechanistic insights from animal studies suggest that fibrosis can be prevented or treated, while human data are so far very limited," comments Karin Sipido, lead investigator of the study. The authors thus designed a study to characterize human cardiac myofibroblasts involved in late-stage $\mathrm{HF}$, and to examine whether their phenotype in end-stage HF can be reversed.

Fibroblasts were isolated from explanted hearts from transplant recipients with end-stage HF $(n=27)$ and without HF ( $n=17)$. The number of myofibroblasts was higher in end-stage HF hearts than in non-HF hearts, and the majority of fibroblastic cells in the end-stage HF hearts were myofibroblasts, some of which remained proliferative. Importantly, these myofibroblasts had a gene expression profile that was associated with a high turnover of matricellular proteins, and expressed high levels of pro-fibrotic cytokines. Inhibition of transforming growth factor- $\beta$ signalling in end-stage-HF myofibroblasts in vitro promoted their dedifferentiation to a less activated state.

Taken together, these in vitro data highlight the potential reversibility of the myofibroblast phenotype in late-stage HF. "We will further explore the molecular signalling pathways that determine differentiation and dedifferentiation of human cardiac fibroblasts, distinguishing those that are responsible for scar and those for interstitial fibrosis," concludes Sipido.

Karina Huynh

ORIGINAL ARTICLE Nagaraju, C. K. et al. Myofibroblast phenotype and reversibility of fibrosis in patients with end-stage heart failure.J. Am. Coll. Cardiol. 73, 2267-2282 (2019) FURTHER READING Saucerman, J. J. et al. Mechanical regulation of gene expression in cardiac myocytes and fibroblasts. Nat. Rev. Cardiol. 16, 361-378 (2019) | Tallquist, M. D. \& Molkentin, J. D. Redefining the identity of cardiac fibroblasts. Nat. Rev. Cardiol. 14, 484-491 (2017)

\title{
Cilia defects linked to mitral valve prolapse
}

The causes of mitral valve prolapse (MVP), a common congenital defect that can lead to arrhythmias, heart failure and sudden cardiac death, are not well understood. A new study now reveals that this condition can be caused by defects in primary cilia. Primary cilia are microtubule-based structures that project from the cell and function as sensory organelles and signalling platforms. Toomer et al. show that mutations in genes associated with primary cilia development and cilia-regulated signalling can cause familial and sporadic nonsyndromic MVP.

First, the researchers studied the involvement of primary cilia in cardiac valve development in mice. They found that these structures varied spatially and temporally during vavulogenesis, mostly disappearing from valve cells after birth, and that primary cilia regulated extracellular matrix (ECM) deposition in valve development. Loss of primary cilia in mice caused valve developmental defects characterized by ECM expansion and altered tissue architecture that led to MVP in adulthood.

Next, Toomer et al. assessed the involvement of primary cilia gene mutations in patients with MVP by performing a gene-set enrichment analysis in a cohort of patients from previous genome-wide association studies. They observed a significant enrichment of MVP-associated variants in the cilia gene set. Further genetic analysis of a large family with inherited, autosomal dominant, nonsyndromic MVP identified a deleterious missense mutation in DZIP1, which encodes a protein involved in the regulation of ciliogenesis and cilia signalling. The pathogenicity of the DZIP1 variant was then confirmed in mice; mice harbouring the DZIP1 familial mutation had impaired ciliogenesis during development that progressed to MVP in adulthood.

Together, these findings reveal a developmental basis for MVP, caused by altered primary cilia-mediated regulation of ECM deposition, and suggest that mutations in primary cilia genes can be the cause of MVP in some patients.

Irene Fernández-Ruiz

ORIGINAL ARTICLE Toomer, K. A. et al. Primary cilia defects causing mitral valve prolapse. Sci. Transl Med. 11, eaax0290 (2019)

\section{ARRHYTHMIAS}

\section{Gene therapy to inhibit CaMKII in CPVT}

Cardiomyocyte-selective gene therapy to inhibit $\mathrm{Ca}^{2+} /$ calmodulin-dependent protein kinase II (CaMKII) effectively suppresses ventricular arrhythmias in mice with mutations known to cause catecholaminergic polymorphic ventricular tachycardia (CPVT). "Delivery of a CaMKII inhibitory peptide by adeno-associated virus (AAV) represents a novel single-dose gene therapy for CPVT," summarize the researchers.

A characteristic feature of CPVT is adrenergically triggered arrhythmias. Inhibition of CaMKII, an adrenergically activated kinase, has been identified as a therapeutic approach for CPVT. However, given that CaMKII is essential for brain function, CaMKII must be selectively inhibited in cardiomyocytes. Therefore, the researchers designed an $A A V$ vector containing a potent $\mathrm{CaMKII}$ inhibitory peptide (AIP) fused to green fluorescent protein (GFP) and expressed from a cardiomyocyte-selective promoter (AAV9-AIP-GFP).

Systemic delivery of AAV9-AIP-GFP to neonatal mice with a CPVT-causing mutation $\left(\right.$ Ryr $\left.^{\mathrm{R} 176 \mathrm{Q} /+}\right)$ resulted in robust expression in the heart, without substantial expression in extracardiac tissues. Ventricular arrhythmias induced by either programmed ventricular pacing or $\beta$-adrenergic stimulation were suppressed. Similar results were obtained in adolescent mice with a CPVT-causing mutation.

Of note, when a cell-permeable form of AIP was delivered directly to human induced pluripotent stem cell-derived cardiomyocytes derived from two patients with different combinations of CPVT-associated amino acid substitutions in RYR2 (one with S404R and N658S and the other with G3946S and $\mathrm{G} 1885 \mathrm{E}$ ), abnormal $\mathrm{Ca}^{2+}$-release events were suppressed. "CaMKII inhibition is a potentially translatable and effective therapy for CPVT patients with diverse RYR2 mutations," conclude the investigators.

Gregory B. Lim

ORIGINAL ARTICLE Bezzerides, V. J. et al. Gene therapy for catecholaminergic polymorphic ventricular tachycardia by inhibition of $\mathrm{Ca}^{2+} /$ calmodulin-dependent kinase II. Circulation https://doi.org/10.1161/CIRCULATIONAHA.118.038514 (2019)

FURTHER READING Bongianino, R. \& Priori, S. G. Gene therapy to treat cardiac arrhythmias. Nat. Rev. Cardiol. 12, 531-546(2015) 